\title{
NR. 08 23.05.2006
}

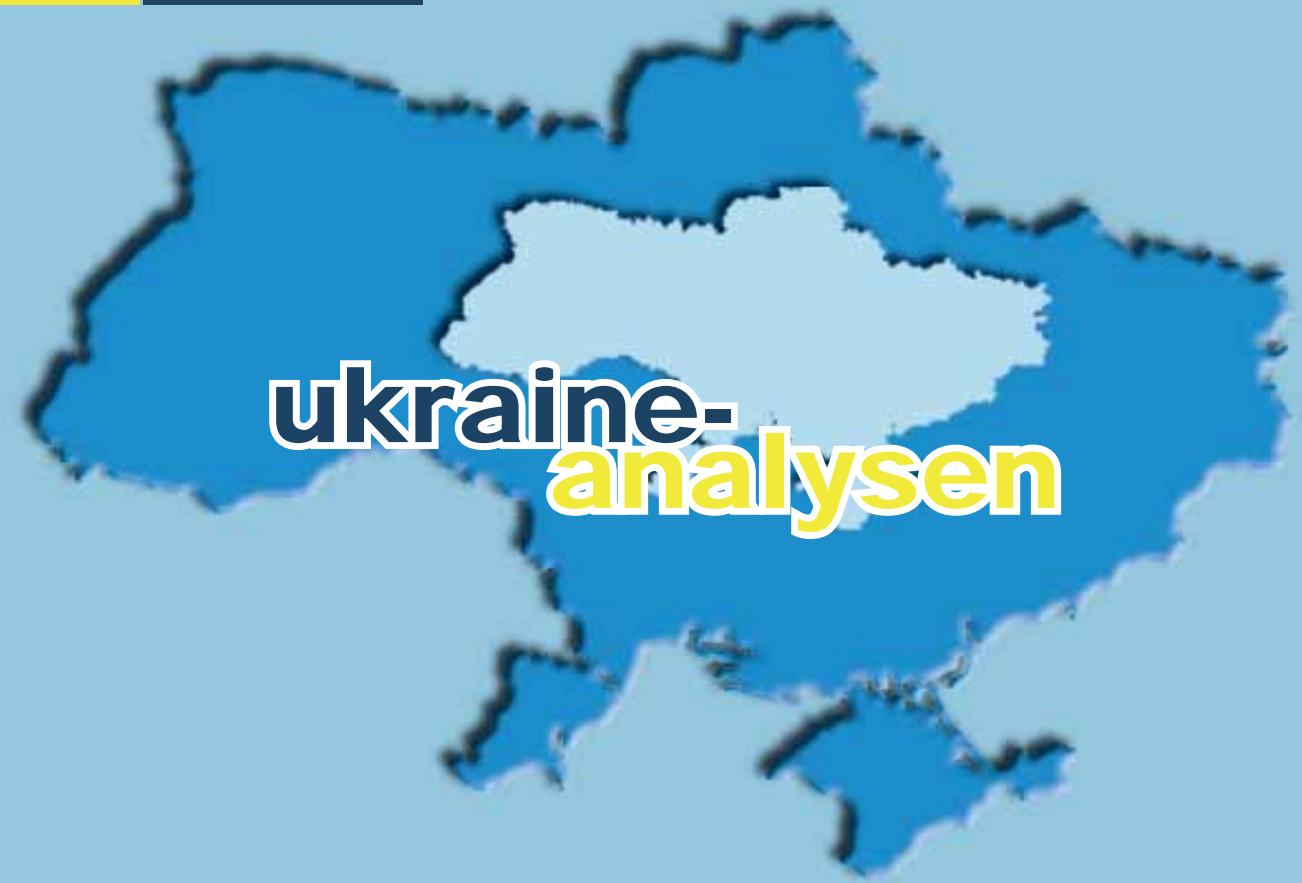

ukraine-analysen.de

\section{DIE PRIVATISIERUNGSAUKTIONEN IN DER UKRAINE}

ANALYSE

Privatisierungsauktionen zwischen Manipulationen, Skandalen und Renationalisierung.

Heiko Pleines, Bremen

TABELLEN UND GRAFIKEN ZUM TEXT

Zahlen und Fakten zur Privatisierung

DOKUMENTATION

Schleuderpreise?

PORTRAITS

Die an Privatisierungsauktionen beteiligten Holdings

CHRONIK

Vom 9. bis 22. Mai 2006

Die nächste Ausgabe der Ukraine-Analysen erscheint am 13.6.2006. Thema wird die Entwicklung der ukrainischen Wirtschaft sein. 


\title{
Analyse
}

\section{Privatisierungsauktionen zwischen Manipulationen, Skandalen und Renationalisierung}

\author{
Von Heiko Pleines, Forschungsstelle Osteuropa, Bremen
}

\section{Zusammenfassung}

Manipulierte Privatisierungsauktionen waren ein wesentlicher Kritikpunkt an Präsident Leonid Kutschma. Sie wurden als Ausdruck des Ausverkaufs staatlichen Vermögens an die Oligarchen gesehen. Die Vertreter des orangen Lagers forderten deshalb eine Revision der Auktionen. Das Ergebnis ist bisher aber bescheiden. Nur zwei der großen Privatisierungsauktionen wurden annuliert. Anstelle einer systematischen juristischen Aufarbeitung stehen politische Querelen. Das Privatisierungsprogramm ist darüber praktisch zum Stillstand gekommen.

\section{Einleitung}

Wit dem Ende der sozialistischen Planwirtschaft Bestandteil marktwirtschaftlicher Reformen begriffen. In der Ukraine begann eine ernsthafte Privatisierung erst Mitte der 1990er Jahre und machte auch in den folgenden Jahren im Vergleich zu anderen post-sozialistischen Ländern nur langsame Fortschritte. Zentrale Kennzahlen zum Umfang und zur Dynamik der Privatisierung zeigt Tabelle 1 auf Seite 7.

Genau wie in Russland entwickelte auch in der Ukraine die Regierung Pläne, große Staatsbetriebe über Auktionen zu privatisieren, um Einnahmen für den Staatshaushalt zu erhalten und strategische Investoren für die Betriebe zu gewinnen. Gegen die Privatisierungsauktionen sprach sich in beiden Ländern die Parlamentsmehrheit aus, die einen Ausverkauf des Staatsvermögens befürchtete.

Der russische Präsident Boris Jelzin umging das Parlament, indem er die Privatisierungsauktionen Mitte 1995 in einem Präsidialerlass regelte. In der Ukraine hingegen gelang es dem Parlament aufgrund seiner stärkeren Stellung im legislativen Prozess, die Kapitalprivatisierung zu blockieren. Erst mit der Bildung einer pro-präsidentiellen Parlamentsmehrheit Anfang 2000 begann deshalb die Privatisierung größerer Staatsbetriebe über Auktionen. Mit der rechtlichen Regelung wurden sowohl in Russland als auch in der Ukraine Auktionen zur wichtigsten Privatisierungsform für große Staatsbetriebe. In der Ukraine hatten die großen Auktionen von 2000 bis 2005 einen Anteil von über $80 \%$ an den gesamten Privatisierungseinnahmen.

Zentrale Akteure der großen ukrainischen Privatisierungsauktionen wurden nationale Finanzinvestoren, sogenannte Oligarchen, die ihre
Akquisitionen in (teilweise informellen) Holdings zusammenfassten. Daneben beteiligten sich in einigen Fällen auch ukrainische und russische Großunternehmen an Auktionen von Staatsbetrieben aus ihrer Branche. Dies gilt insbesondere für die Erdölund Metallindustrie. Ausländische Investoren aus anderen Ländern fanden nur in wenigen Ausnahmefällen Zugang zu großen Privatisierungsauktionen. Einen Überblick über die Auktionen gibt Tabelle 2 auf Seite 7. Kurzportraits der beteiligten Holdings ukrainischer Oligarchen finden sich auf Seite 11.

Durch ihre Teilnahme an den Privatisierungsauktionen erlangten die erfolgreichen ukrainischen Holdings die Kontrolle über einige der größten ukrainischen Unternehmen. Ende 2004 hielten sie so Anteile an 22 der 100 größten ukrainischen Unternehmen, an 15 davon besaßen sie die Mehrheit.

\section{Die Netzwerke der Oligarchen unter Kutschma}

as politische Kalkül von Präsident Kutschma konzentrierte sich nicht auf die schwach entwickelten politischen Lager, wie sie im Parlament vertreten waren, sondern auf regionale Seilschaften. Vertreter der politischen Elite einer Region wurden in nationale Ämter nach Kiew berufen. Mit Hilfe ihrer neu gewonnenen Kompetenzen konnten diese Politiker dann die Unternehmer fördern, mit denen sie durch Seilschaften verbunden waren. Die Politiker scheinen dafür in der Regel am Gewinn der Unternehmer über politische Korruption beteiligt worden zu sein. Die regionalen Seilschaften nutzten ihren Einfluss auf Massenmedien und auf die politische Stimmung in ihrer Region, um die öffentliche Meinung zugunsten des Präsidenten zu beeinflussen.

Auf diese Weise profitierten alle Beteiligten vom 
Arrangement. Die regionalen Politiker erhielten einflussreiche Positionen auf der nationalen Ebene und Bestechungszahlungen der Unternehmer. Die Unternehmer erhielten eine Vorzugsbehandlung durch die Politik, die ihnen immense Gewinne bescherte. Und der Präsident erhielt Unterstützung für seinen Wahlkampf. Entscheidend für den Präsidenten war nun, dass er so viele regionale Seilschaften integrierte, dass deren vereinigte Wahlkampfhilfe ausreichte, um die Präsidentschaftswahl zu gewinnen.

Gleichzeitig musste der Präsident seine Position als zentraler Vermittler in diesem System sichern, um das Aufkommen eines internen Konkurrenten um das Präsidentenamt zu vermeiden. Dies hatte zur Folge, dass hohe Ämter häufig umbesetzt wurden, um keinen Konkurrenten heranwachsen zu lassen und durch regelmäßige Umverteilungen alle regionalen Seilschaften bedienen zu können. Klassisches Beispiel hierfür ist das Amt des Ministerpräsidenten. In der zehnjährigen Amtszeit von Präsident Kutschma amtierten sieben Ministerpräsidenten. Die Mehrheit von ihnen kam aus Dnipropetrowsk und gehörte damit zur regionalen Seilschaft Kutschmas. Zum Anfang der Amtszeit Kutschmas stellten alte sowjetische Seilschaften den Ministerpräsidenten. Gegen Ende der zweiten Amtszeit gelang einer Seilschaft aus Donezk unter Viktor Janukowitsch der Zugriff auf das Amt des Ministerpräsidenten. Der einzige Ministerpräsident ohne Verbindungen zu einer einflussreichen Seilschaft war der reformorientierte Viktor Juschtschenko von Ende 1999 bis Frühjahr 2001.

In diesem Sinne scheinen Seilschaften auch Kontrolle über den Fonds für Staatsvermögen benutzt zu haben, um ihnen nahe stehende Unternehmen zu bevorzugen. Versuche des privatisierungskritischen Parlaments, Einfluss über den Fonds zu gewinnen, scheiterten 1998 an einem Verfassungsgerichtsurteil. Der Fonds untersteht dem Präsidenten. Der Leiter des Fonds wird vom Präsidenten mit Zustimmung des Parlaments ernannt und entlassen.

Leiter des Fonds war von August 1998 bis April 2003 Alexander Bondar. Er hatte seine Karriere bereits in der Sowjetunion in der Kiewer Lokalverwaltung begonnen und wechselte 1998 von der lokalen Kiewer Verwaltung für Staatsvermögen in die nationale Struktur. Von seiner Karriere her stand er weder der Dnipropetrowsker noch der Donezker Seilschaft nahe. Bereits 1999 erhielt er aber mit Michael Tschetschetow einen Stellvertreter mit enger Bindung an das Janukowitsch-Lager. Tschetschetow, ein Wirtschaftsingenieur aus Charkiw, war Mitte der 1990er als Mitglied der Liberalen Partei Abgeordneter der Werchowna Rada für einen Donezker Wahlkreis. Mit dem Aufstieg von Janukowitsch schloss er sich jedoch der von Janukowitsch initiierten Partei der
Regionen an, in deren Präsidium er mit Janukowitsch zusammenarbeitete. Nach der Entlassung Bondars im April 2003 übernahm Tschetschetow die Leitung des Fonds für Staatsvermögen, die er bis zum Ende der Amtszeit von Janukowitsch behielt.

\section{Oligarchenparteien im Parlament}

Cegen Ende der 1990er Jahre hatten die regioInalen Seilschaften ihre Struktur und ihre Verbindungen zur nationalen Exekutive gefestigt. Diese Stabilität war aber abhängig von der Person des Präsidenten. Hieraus ergaben sich für die regionalen Seilschaften Ende der 1990er Jahre zwei zentrale Probleme. Erstens musste die Wiederwahl Kutschmas bei den Präsidentschaftswahlen im Herbst 1999 gesichert werden. Zweitens konnte der Präsident als Leiter der Exekutive den regionalen Seilschaften zwar erhebliche Vorteile verschaffen, seine Handlungsmöglichkeiten wurden aber durch die Opposition des Parlaments erheblich eingeschränkt.

Beide Probleme legten die Gründung von Parteien nahe. Mehr noch als Massenmedien konnten Parteien dazu dienen, Wähler für den Präsidenten zu mobilisieren. Gleichzeitig konnten neue Parteien im Parlament die Opposition zum Präsidenten aufbrechen. Über die Schaffung einer präsidentennahen Mehrheit im Parlament konnten die regionalen Seilschaften also zum einen Kutschma einen politischen Dienst erweisen, der seine Gunst auch über den erfolgreichen Wahlkampf hinaus sicherte. Zum anderen bekamen sie damit neben dem Zugang zu Regierungsämtern einen zweiten Kanal, über den sie politische Entscheidungsprozesse direkt im eigenen Interesse gestalten konnten. Hinzu kam die Tatsache, dass die Immunität der Parlamentsabgeordneten vor Strafverfolgung für viele Unternehmer eine Rückversicherung gegen Korruptionsvorwürfe darstellte.

Die Kutschma-nahen Unternehmerparteien bewirkten Ende 1999 eine deutliche Kräfteverschiebung im politischen System der Ukraine. Durch einen gut finanzierten Wahlkampf sicherten sie die Wiederwahl von Präsident Kutschma. Gleichzeitig warben sie im Parlament in großem Umfang Abgeordnete oppositioneller Fraktionen ab. Anfang 2000 stellten die pro-präsidentiellen Parteien erstmals eine Mehrheit im Parlament, die sie nutzten, um Vertreter der linken Opposition aus ihren Ämtern zu verdrängen. Damit erlangten sie eine zentrale Position im legislativen Prozess und konnten unter anderem auch die Privatisierungsgesetze beeinflussen.

\section{Die rechtliche Regelung der Auktionen}

Mit der Schaffung einer pro-präsidentiellen Mehrheit im Parlament wurde es im Frühjahr 
2000 möglich, das Gesetz über das staatliche Privatisierungsprogramm zu verabschieden, das eine Beschleunigung der Privatisierung über Auktionen vorsah. Die Regelung der Privatisierungsauktionen wurde also von den unternehmerdominierten Parteien durch das Parlament gebracht. Das Privatisierungsprogramm 2000 hatte ursprünglich eine Laufzeit von zwei Jahren. Die Verabschiedung eines Nachfolgeprogramms gelang jedoch nicht, so dass das Privatisierungsprogramm 2000 bis zum Jahresende 2004 die Grundlage der Privatisierung blieb.

Für die ukrainischen Holdings waren bei der Regelung der Privatisierungsauktionen drei Aspekte von zentraler Bedeutung:

Erstens wurden ausländische Bieter diskriminiert. Das Privatisierungsprogramm räumte strategischen Investoren ausdrücklich Vorrang bei der Privatisierung von Großbetrieben ein. Strategische Investoren wurden dabei definiert als in- oder ausländische Investoren, die über nicht weniger als drei Jahre Erfahrung auf dem relevanten Markt verfügen und an der Aufrechterhaltung des Marktanteils des zu privatisierenden Unternehmens interessiert sind. Bei vielen Auktionen wurde der relevante Markt als ukrainischer Markt definiert, was die meisten ausländischen Investoren mangels Engagement in der Ukraine ausschloss. Innerhalb der Ukraine besaßen aber nur die Holdings die erforderliche Finanzkraft zur Teilnahme an großen Privatisierungsauktionen.

Zweitens schufen sich die für die Privatisierungsauktionen zuständigen Organe der staatlichen Exekutive erhebliche Spielräume für Manipulationen. Eine allgemeine Regelung zu Interessenkonflikten bei den für die Privatisierungsauktionen zuständigen Kommissionsmitgliedern wurde so nicht getroffen. Die Informationspflicht der Kommission wurde nicht konkretisiert. Die Kommission war zum Beispiel nicht verpflichtet, den unterlegenen Auktionsteilnehmern eine Begründung für ihre Entscheidung zu geben. Die Mitglieder der für die Privatisierungsauktionen zuständigen Kommissionen besaßen somit de facto keine Rechenschaftspflicht. Außerdem bestand die Möglichkeit, durch die rechtliche Regelung einer konkreten Privatisierungsauktion bereits im Vorfeld bestimmten Bietern eine Vorzugsbehandlung zu gewähren. Für die Privatisierung der Holdinggesellschaft Ukrrudprom, die die ukrainische Eisenerzproduktion zusammenfasste, wurde so ein spezielles Gesetz erlassen, dass ausdrücklich vorsah, dass Bietern, die bereits Anteile an Ukrrudprom besaßen, bei der weiteren Privatisierung Vorrang einzuräumen sei.

Gleichzeitig wurde die Ausschreibung von Privatisierungsauktionen häufig so angesetzt, dass nur vorab informierte Bieter die Möglichkeit be- saßen, fristgerecht ein Angebot vorzubereiten. Die Ausschreibungen für Privatisierungsauktionen wurden so lange Zeit nur im Amtsblatt sowie auf der Internetseite des Fonds für Staatsvermögen veröffentlicht. Die Frist bis zur Angebotsabgabe betrug dabei im Extremfall nur wenige Wochen, was nicht ausreichte, um den Wert des angebotenen Unternehmens abzuschätzen, einen Investitionsplan zu entwickeln und bei Bedarf ein Konsortium zur Gebotsabgabe zu bilden.

Verstärkt wurde die Bevorzugung von Insidern noch durch die Möglichkeit, private Firmen zu Verwaltern des Staatsanteils an zu privatisierenden Unternehmen zu ernennen. Häufig wurden Vertreter der Holdings zu Verwaltern von Staatsanteilen ernannt. Sie hatten so gegenüber konkurrierenden Bietern bei den Privatisierungsauktionen einen Informationsvorsprung. Außerdem konnten sie über gezielte Investitionen, deren Weiterführung in die Auktionsbedingungen aufgenommen wurde, oder über Kreditvergabe, die ihnen als Gläubiger ein Mitspracherecht beim Verkauf sicherte, erheblichen Einfluss auf den Verlauf der Auktion nehmen.

Im Ergebnis besaß die Exekutive die Möglichkeit, ausländische Bieter auszuschließen, einen bestimmten (in- oder ausländischen) Bieter durch Vorabinformationen und die Ernennung zum Verwalter des zu privatisierenden Staatsanteils erheblich zu bevorteilen und anschließend in einem intransparenten Verfahren den Sieger weitgehend unkontrolliert festzulegen.

Drittens wurde der Mindestpreis für die angebotenen Unternehmensanteile oft relativ niedrig angesetzt. Ein Regierungsdekret vom Mai 2002 erlaubte es dem Fonds für Staatsvermögen dann, bei unzureichender Teilnahme an der Auktion das Mindestgebot um bis zu 30\% zu senken. Aufgrund der Manipulationsmögli chkeiten der Exekutive kam es nur in Ausnahmefällen zu echtem Wettbewerb, der zu Geboten deutlich über dem Mindestpreis führte. Ab Herbst 2002 wurden Unternehmen so zu Preisen verkauft, die in Relation zu ihrem Gewinn, extrem günstig waren. Der Preis für das Eisenerzwerk Piwdennij, das im September 2002 verkauft wurde, betrug so anteilig gerade mal etwas mehr als den zweifachen Jahresgewinn. Die Eisenerzwerke Centralnij und Piwnitschnij wurden 2004 gar für nur einen anteiligen Jahresgewinn verkauft. Und das große Stahlwerk Kryvorizhstal wurde im selben Jahr für etwa drei Jahresgewinne veräußerst. Siehe dazu die Dokumentation auf Seite 9.

\section{Der Verlauf der Auktionen}

ie großen ukrainischen Privatisierungsauktionen, die von 2000 bis 2004 stattfanden, lassen sich in zwei Phasen einteilen. (Einen Überblick über die 
Auktionen gibt Tabelle 2 auf Seite 7.)

In der ersten Phase, von März 2000 bis April 2001, war Viktor Juschtschenko Ministerpräsident und Alexander Bondar Leiter des Fonds für Staatsvermögen. In dieser Phase fanden insgesamt fünf große Privatisierungsauktionen statt, die dem Staat Einnahmen von 0,4 Mrd. US-Dollar brachten. In einer Übergangsphase unter Ministerpräsident Kinach wurden dann nur drei Unternehmen für zusammen weniger als 0,1 Mrd. US-Dollar über größere Privatisierungsauktionen verkauft. In der zweiten Phase der großen Privatisierungsauktionen, von April 2003 bis Juli 2004, war dann Viktor Janukowitsch Ministerpräsident und sein politischer Partner Michael Tschetschetow wurde Leiter des Fonds für Staatsvermögen. In dieser Phase wurden zehn große Privatisierungsauktionen erfolgreich abgeschlossen und erbrachten Einnahmen von insgesamt 1,6 Mrd. US-Dollar.

Bezüglich der erfolgreichen Bieter unterscheiden sich die beiden Phasen eklatant. Unter Juschtschenko / Bondar gewannen ausländische Bieter alle der großen Privatisierungsauktionen. In der Hälfte der Fälle handelt es sich dabei um große russische Unternehmen. Unter Janukowitsch / Tschetschetow verkehrte sich das Verhältnis. Nur bei einer Auktion gewann ein ausländischer Bieter, eine russische Firma. Alle anderen Auktionen wurden von ukrainischen Holdings gewonnen.

Auch bezüglich der erzielten Preise unterscheiden sich die beiden Phasen deutlich. Unter Juschtschenko / Bondar wurden vor allem verlustbringende Unternehmen verkauft. Durch internationalen Wettbewerb wurden dabei in der Regel Preise erzielt, die deutlich über den angesetzten Mindestpreis hinausgingen. Unter Janukowitsch / Tschetschetow wurden hingegen gewinnbringende Unternehmen häufig zu vergleichsweise günstigen Preisen verkauft, wobei höhere Gebote von ausländischen Interessenten teilweise nicht zugelassen wurden. Siehe dazu die Dokumentation auf Seite 9.

Zusammenfassend kann so konstatiert werden, dass Juschtschenko als Ministerpräsident versuchte, den Einfluss ukrainischer Finanzinvestoren einzuschränken und deshalb ausländische Bieter zu einer Teilnahme an Privatisierungsauktionen motivierte. Gleichzeitig übte er politischen Druck aus, um den Fonds für Staatsvermögen zur Akzeptanz dieser Strategie zu bewegen. Er kritisierte so öffentlich Entscheidungen des Fonds für konkrete Bieter und zeigte Manipulationen auf. Janukowitsch hingegen brachte mit Tschetschetow seinen eigenen Mann an die Spitze des Fonds für Staatsvermögen. Im Ergebnis wurden mit SCM und ISD die Holdings aus seiner Donezker Seilschaft bevorzugt. Zusätzlich wurden die Interessen der Präsident Kutschma nahe stehenden Interpipe-Holding berücksichtigt. Der Privatbank hingegen gelang es trotz mehrerer Gebote nicht, eine große Auktion zu gewinnen.

\section{Renationalisierung unter Präsident Juschtschenko?}

ie Opposition gegen Präsident Kutschma protestierte heftig gegen die manipulierten Privatisierungsauktionen, die sie sowohl als Ausdruck der Korruptionsnetzwerke zwischen Politik und Oligarchen als auch als Ausverkauf staatlichen Vermögens sahen. Als nach der „Orangen Revolution“ Viktor Juschtschenko Präsident und Julia Timoschenko Ministerpräsidentin wurden, versprachen sie eine Revision der Privatisierungsauktionen. Während Präsident Juschtschenko etwa 30 Auktionen überprüfen lassen wollte, stellten Regierungsmitglieder mehrfach grundsätzlich alle bisherigen Privatisierungen in Frage. Timoschenko kündigte gar eine Reprivatisierung von 3.000 Unternehmen an. Auch unterlegene Bieter aus den Privatisierungsauktionen nutzten diese Chance, um vor Gericht gegen Auktionsergebnisse zu klagen. Im Ergebnis kam auf die Gerichte eine Klagewelle zu. Im ersten Halbjahr 2005 registrierte das Innenministerium über 1.700 staatsanwaltschaftliche Ermittlungen wegen Privatisierungsvergehen.

Die Diskussion um die Privatisierungsauktionen führte so im Frühjahr 2005 zu einer politischen Krise. Gleichzeitig gefährdete die breite Debatte um Renationalisierungen das Investitionsklima. Praktisch stellte sich das Problem, dass Gesetzesverstöße, die formal eine richterliche Annullierung der Privatisierungsauktionen begründen könnten, nur schwer zu belegen sind, da die Regeln für die Auktionen bewusst legale Manipulationsmöglichkeit en und Schlupflöcher geschaffen hatten.

Im Ergebnis wurden bisher von den großen Privatisierungsauktionen nur zwei durch Gerichtsbeschluss annulliert. Im Sommer 2005 wurde der privatisierte Anteil an Kryvorizhstal gegen Zahlung des Kaufpreises an den Staat zurückgegeben. Kryvorizhstal wurde dann im Oktober auf einer Auktion reprivatisiert. Diskriminierende Regeln für ausländische Investoren wurden abgeschafft und die Auktion wurde ergebnisoffen und transparent organisiert. Stattdessen wurden die Auflagen bezüglich Mode rnisierungsinvestitionen und sozialer Verpflichtungen erhöht. Im Ergebnis lag der Verkaufspreis mit 4,8 Mrd. US-Dollar etwa sechsmal höher als bei der vorhergehenden Auktion.

Nach langwierigen Berufungsverfahren wurde im Frühjahr 2006 auch der 2003 in zwei Privatisierungsauktionen veräußerte Anteil am Eisenlegierungswerk Nikopol an den Staat zurück- 
gegeben. Das Parlament stimmte jedoch gegen eine Reprivatisierung des Werkes.

Im Ergebnis lässt sich festhalten, dass eine systematische Überprüfung der Privatisierungsauktionen nicht stattgefunden hat. Die beiden bisher erfolgten Annullierungen sind eher Gegenstand politischer Kontroversen als richterlicher Tatbestandserfassungen. Gleichzeitig ist der Privatisierungsprozess in der Ukraine wieder ins Stocken geraten.

Ohne die Kryvorizhstal-Auktion betrugen die Privatisierungseinnahmen 2005 geradeeinmal0,2 Mrd. US-Dollar, was der niedrigste Stand seit 2002 gewesen wäre. Die einzige große Auktion war dabei der Verkauf der verbliebenen $40 \%$ Staatsanteil am Kohlebergwerk Krasnodonvuhillya. Interessanterweise gewann hier mit einer Tochterfirma von SCM, der Holding des Donezker Unternehmers Rinat Achmetow, derselbe Bieter wie bei der vorhergehenden Auktion von 60\% an Krasnodonvuhillya unter Präsident Kutschma.

Ein im August 2005 von der Regierung verabschiedeter Plan zur Beschleunigung der Privatisierung scheiterte dann an den politischen Turbulenzen im Zuge der Regierungskrise nach der Entlassung Timoschenkos. Während der im Herbst von der Regierung erstellte Entwurf für den Staatshaushalt 2006 noch Privatisierungseinnahmen von gut 1,5 Mrd. US-Dollar vorsah, waren es in der endgültigen Version vom Januar 2006 nur noch 0,4 Mrd. US-Dollar. Das Privatisierungsprogramm ist damit praktisch zum Stillstand gekommen. Sowohl für die betroffenen Unternehmen als auch für den ukrainischen Staatshaushalt wäre ein transparentes Privatisierungsprogramm aber deutlich hilfreicher als eine Schlammschlacht um juristisch in der Regel nicht eindeutig belegbare Privatisierungsvergehen.

Über den Autoren:

Dr. Heiko Pleines ist wissenschaftlicher Mitarbeiter an der Forschungsstelle Osteuropa in Bremen.

Lesetipp:

Heiko Pleines: Ukrainische Seilschaften. Informelle Einflussnahme in der ukrainischen Wirtschaftspolitik 1992-2004,

Münster (LIT) 2005

Tabellen und Grafiken zum Text

\section{Zahlen und Fakten zur Privatisierung}

Grafik 1: Staatseinnahmen aus der Privatisierung 1992-2005 (in Mrd. US-Dollar)

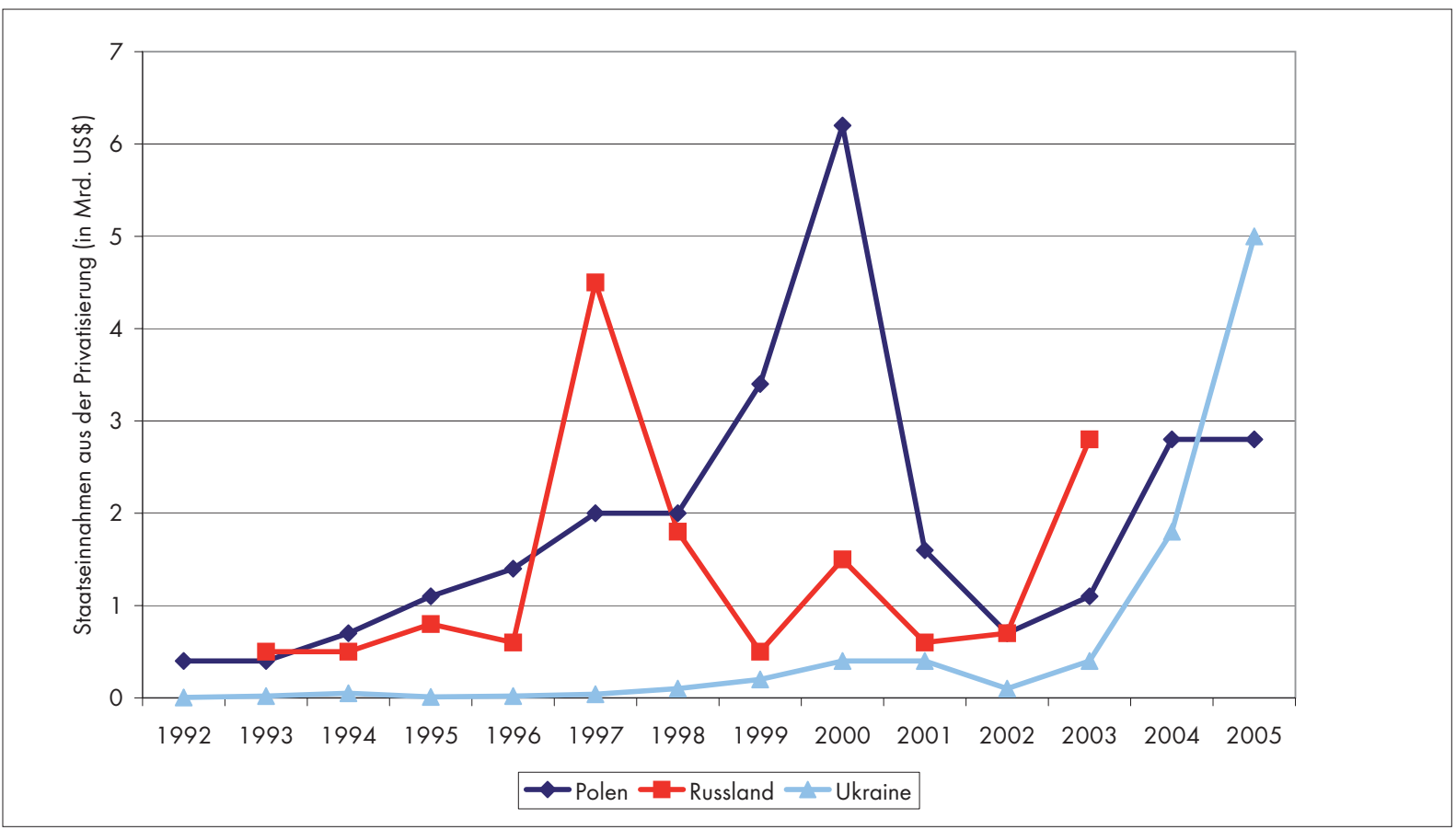


Tabelle 1: Kennzahlen zum Verlauf der ukrainischen Privatisierung 1992-2005

\begin{tabular}{|l|c|c|c|c|c|c|c|} 
& $\mathbf{1 9 9 2}$ & $\mathbf{1 9 9 3}$ & $\mathbf{1 9 9 4}$ & $\mathbf{1 9 9 5}$ & $\mathbf{1 9 9 6}$ & $\mathbf{1 9 9 7}$ & $\mathbf{1 9 9 8}$ \\
\hline $\begin{array}{l}\text { Staatseinnahmen aus der Privatisierung } \\
\text { (in Mrd. US\$) }\end{array}$ & 0,005 & 0,02 & 0,05 & 0,01 & 0,02 & 0,04 & 0,1 \\
\hline Anteil an den gesamten Staatseinnahmen & $0,1 \%$ & $0,2 \%$ & $0,3 \%$ & $0,1 \%$ & $0,1 \%$ & $0,2 \%$ & $1,0 \%$ \\
\hline Anteil am BIP & $0,02 \%$ & $0,06 \%$ & $0,1 \%$ & $0,03 \%$ & $0,04 \%$ & $0,08 \%$ & $0,4 \%$ \\
\hline Zahl der privatisierten Betriebe & 30 & 3.555 & 8.249 & 16.211 & 19.434 & 8.494 & 5.371 \\
\hline Anteil der Privatwirtschaft am BIP & $10 \%$ & $15 \%$ & $40 \%$ & $45 \%$ & $50 \%$ & $55 \%$ & $55 \%$ \\
\hline $\begin{array}{l}\text { Staatseinnahmen aus der Privatisierung } \\
\text { (in Mrd. US\$) }\end{array}$ & $\mathbf{1 9 9 9}$ & $\mathbf{2 0 0 0}$ & $\mathbf{2 0 0 1}$ & $\mathbf{2 0 0 2}$ & $\mathbf{2 0 0 3}$ & $\mathbf{2 0 0 4}$ & $\mathbf{2 0 0 5}$ \\
\hline Anteil an den gesamten Staatseinnahmen & $1,6 \%$ & 0,4 & 0,4 & 0,1 & 0,4 & 1,8 & 5,0 \\
\hline Anteil am BIP & $0,5 \%$ & $1,2 \%$ & $1,0 \%$ & $0,3 \%$ & $0,8 \%$ & $2,8 \%$ & $6,1 \%$ \\
\hline Zahl der privatisierten Betriebe & 5.177 & 5.201 & 6.023 & 6.150 & 6.546 & 1.106 & 5.664 \\
\hline Anteil der Privatwirtschaft am BIP & $55 \%$ & $60 \%$ & $60 \%$ & $65 \%$ & $65 \%$ & $65 \%$ & $65 \%$ \\
\hline
\end{tabular}

Anmerkung: Die Umrechnung von nationaler Währung in US-Dollar erfolgte jeweils zum Jahresmittelkurs.

Quellen: Poslannja Presidenta Ukrajiny do Werchovnoi Radi Ukrajiny, Kiew 2004, S. 188-191, Fond Dershawnoho majna Ukrajiny (Ukrainischer Fond für Staatsvermögen, uww.spfugov.ua), Institute for Economic Research and Policy Consulting (www.ier.kiev.ua).

Anteil der Privatwirtschaft nach EBRD Transition Report, London, laufende Jahrgänge.

Tabelle 2: Die großen ukrainischen Privatisierungsauktionen 2000 - 2005

\begin{tabular}{|l|l|l|l|l|}
\hline Datum & $\begin{array}{l}\text { Verkaufter } \\
\text { Aktienanteil }\end{array}$ & Branche & Bedingungen & $\begin{array}{l}\text { Bieter - Gebot [in Mio. US\$] } \\
\text { [der erfolgreiche Bieter ist fett hervorgehoben] }\end{array}$ \\
\hline $\begin{array}{l}\text { März } \\
2000\end{array}$ & $\begin{array}{l}\text { Aluminium- } \\
\text { werk } \\
\text { Mykolaiw } \\
(30 \%)\end{array}$ & $\begin{array}{l}\text { Metall- } \\
\text { industrie }\end{array}$ & $\begin{array}{l}\text { Investition von 190 } \\
\text { Mio. US\$. }\end{array}$ & $\begin{array}{l}\text { Ukrainian Aluminum company [Siberian Aluminium } \\
\text { (Russia) - 75\%, Ukrsibbank - 25\%] - 101 } \\
\text { Siberian Aluminium (Russland) - 50 } \\
\text { National Aluminium Corporation -30 } \\
\text { KrAZ (Russland) - disqualifiziert }\end{array}$ \\
\hline $\begin{array}{l}\text { Juli } \\
2000\end{array}$ & $\begin{array}{l}\text { Linos } \\
(67,41 \%)\end{array}$ & $\begin{array}{l}\text { Erdöl- } \\
\text { raffinerie }\end{array}$ & $\begin{array}{l}\text { Schuldenbegleichung } \\
\text { von 5,4 Mio. US\$, } \\
\text { Investitionsprogramm } \\
\text { von 34 Mio. US\$ } \\
\text { sowie Liefergarantie für } \\
\text { Rohöl. }\end{array}$ & $\begin{array}{l}\text { TNK (Russland) - 10 + 5 } \\
\text { Prodintorg [TNK] } \\
\text { Lukoil (Rusland) } \\
\text { Ukrgazbank/Gazprom (Russland) }\end{array}$ \\
$\begin{array}{l}\text { Kazakoil (Kasachstan) } \\
2001\end{array}$ & $\begin{array}{l}\text { Aluminium- } \\
\text { werk } \\
\text { Saporishja } \\
(68,1 \%)\end{array}$ & $\begin{array}{l}\text { Metall- } \\
\text { industrie }\end{array}$ & $\begin{array}{l}\text { Schuldenübernahme } \\
\text { von 81 Mio. US\$ und } \\
\text { Investitionszusage im } \\
\text { Umfang von 200 Mio. }\end{array}$ & $\begin{array}{l}\text { AvtoVAZ (Russland) - 71 + 81 } \\
\text { KrAZ (Russland) - 103 / disqualifiziert } \\
\text { Zwei weitere Bieter }\end{array}$ \\
\hline $\begin{array}{l}\text { April } \\
2001\end{array}$ & $\begin{array}{l}\text { Chersonoblen- } \\
\text { ergo (65\%) } \\
\text { Kyrowohra- } \\
\text { doblenergo } \\
(51 \%) \\
\text { Sewastopol- } \\
\text { miskenergo } \\
(70 \%) \\
\text { Shytomy- } \\
\text { roblenergo } \\
(75,6 \%)\end{array}$ & $\begin{array}{l}\text { Strom- } \\
\text { wirtschaft }\end{array}$ & $\begin{array}{l}\text { Externer Berater: Cre- } \\
\text { dit Suisse First Boston }\end{array}$ & $\begin{array}{l}\text { VEZ (Slowakei) - 91 } \\
\text { UES (Russland) - disqualifiziert } \\
\text { Interessenten, die ihr Gebot zurückziehen: } \\
\text { Bewag (Deutschland), Kansai (Japan), Union Fenosa } \\
\text { (Spanien), Synergy (USA), Poltawaoblenergo }\end{array}$ \\
\hline
\end{tabular}




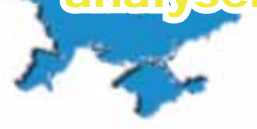

Tabelle 2: Die großen ukrainischen Privatisierungsauktionen 2000 - 2005 (Fortsetzung)

\begin{tabular}{|c|c|c|c|c|}
\hline Datum & $\begin{array}{l}\text { Verkaufter } \\
\text { Aktienanteil }\end{array}$ & Branche & Bedingungen & $\begin{array}{l}\text { Bieter - Gebot [in Mio. US\$] } \\
\text { [der erfolgreiche Bieter ist fett hervorgehoben] }\end{array}$ \\
\hline $\begin{array}{l}\text { April } \\
2001\end{array}$ & $\begin{array}{l}\text { Kyiwoblenergo } \\
(75 \%) \\
\text { Riwneobl- } \\
\text { energo }(75 \%)\end{array}$ & $\begin{array}{l}\text { Strom- } \\
\text { wirtschaft }\end{array}$ & $\begin{array}{l}\text { Externer Berater: } \\
\text { Credit Suisse First } \\
\text { Boston }\end{array}$ & $\begin{array}{l}\text { AES Corp. (USA) - } 69 \\
\text { Electricité de France } \\
\text { Kansai (Japan) } \\
\text { Union Fenosa (Spanien) } \\
\text { VEZ (Slowakei) } \\
\text { Interessenten, die ihr Gebot zurückziehen: } \\
\text { Bewag (Deutschland), Synergy (USA) } \\
\text { Drei Bieter nicht zugelassen: } \\
\text { UES (Russland), Enron (USA), Poltavaselchozenergo }\end{array}$ \\
\hline $\begin{array}{l}\text { Aug. } \\
2001\end{array}$ & $\begin{array}{l}\text { Röhrenwerk } \\
\text { Charzysk } \\
(76 \%)\end{array}$ & $\begin{array}{l}\text { Metall- } \\
\text { industrie }\end{array}$ & $\begin{array}{l}\text { Schuldenübernahme } \\
\text { von } 22 \text { Mio. US\$, } \\
\text { Investitionsprogramm, } \\
\text { Aufrechterhaltung der } \\
\text { Produktion }\end{array}$ & $\begin{array}{l}\text { ISD - } \mathbf{2 3}+\mathbf{2 2} \\
\text { Gazprom (Russland) } \\
\text { Poltavskij GOK - disqualifiziert } \\
\text { Interessenten, die ihr Gebot zurückziehen: } \\
\text { Mannesmann (Deutschland) }\end{array}$ \\
\hline $\begin{array}{l}\text { Jan. } \\
2002\end{array}$ & $\begin{array}{l}\text { AvtoZAZ } \\
(81,59 \%)\end{array}$ & Fahrzeugbau & $\begin{array}{l}\text { Schuldenübernahme } \\
\text { von } 5 \text { Mio. US\$, } \\
\text { Aufrechterhaltung der } \\
\text { Produktion, Arbeits- } \\
\text { platzgarantie für } 3 \\
\text { Jahre. }\end{array}$ & $\begin{array}{l}\text { Ukravto - } \mathbf{1 0}+\mathbf{5} \\
\text { Interessenten, die ihr Gebot zurückziehen: } \\
\text { AvtoVAZ (Russland), ein weiterer Bieter }\end{array}$ \\
\hline $\begin{array}{l}\text { Sept. } \\
2002\end{array}$ & $\begin{array}{l}\text { Eisenerzwerk } \\
\text { Piwdennyj } \\
(35,74 \%)\end{array}$ & $\begin{array}{l}\text { Metall- } \\
\text { industrie }\end{array}$ & & $\begin{array}{l}\text { Ukrainian Metallurgy Company [Ukrsibbank / ISD] } \\
\mathbf{- 1 7} \\
\text { Ukrsibbank } \\
\text { Privat-Intertrading - disqualifiziert } \\
\mathbf{6} \text { weitere Bieter nicht zugelassen }\end{array}$ \\
\hline $\begin{array}{l}\text { April } \\
2003\end{array}$ & $\begin{array}{l}\text { Eisenlegie- } \\
\text { rungswerk } \\
\text { Nikopol } \\
(25 \%)\end{array}$ & $\begin{array}{l}\text { Metall- } \\
\text { industrie }\end{array}$ & strategischer Investor & $\begin{array}{l}\text { Pryndniprowye [Interpipe] - } \mathbf{3 8} \\
\text { keine weiteren Bieter zugelassen }\end{array}$ \\
\hline $\begin{array}{l}\text { Aug. } \\
2003\end{array}$ & $\begin{array}{l}\text { Eisenlegie- } \\
\text { rungswerk } \\
\text { Nikopol } \\
(25 \%)\end{array}$ & $\begin{array}{l}\text { Metall- } \\
\text { industrie }\end{array}$ & strategischer Investor & Pryndniprowye [Interpipe] - 38 \\
\hline $\begin{array}{l}\text { Dez. } \\
2003\end{array}$ & $\begin{array}{l}\text { Dniprope- } \\
\text { trowsker } \\
\text { Metallwerk } \\
\text { „Dsershinskyj“ } \\
(98,81 \%)\end{array}$ & $\begin{array}{l}\text { Metall- } \\
\text { industrie }\end{array}$ & & $\begin{array}{l}\text { ISD - } \mathbf{1 3 3} \\
\text { ein Bieter nicht zugelassen }\end{array}$ \\
\hline $\begin{array}{l}\text { Dez. } \\
2003\end{array}$ & $\begin{array}{l}\text { Dniprope- } \\
\text { trowsker } \\
\text { Metallwerk } \\
\text { „Petrowskyj“ } \\
(42,26 \%)\end{array}$ & $\begin{array}{l}\text { Metall- } \\
\text { industrie }\end{array}$ & $\begin{array}{l}\text { Modernisierung des } \\
\text { Betriebs }\end{array}$ & $\begin{array}{l}\text { ISD - } 12 \\
\text { Privat Intertrading - disqualifiziert }\end{array}$ \\
\hline $\begin{array}{l}\text { Juni } \\
2004\end{array}$ & $\begin{array}{l}\text { Kryworishstal } \\
(93,02 \%)\end{array}$ & $\begin{array}{l}\text { Metall- } \\
\text { industrie }\end{array}$ & $\begin{array}{l}\text { strategischer Investor } \\
\text { mit Produktion in der } \\
\text { Ukraine }\end{array}$ & $\begin{array}{l}\text { IMU [SCM - 56\% + Interpipe - 44\%] - } 799 \\
\text { IG [ISD] } \\
\text { Keine weiteren Bieter zugelassen, darunter: } \\
\text { Severstal (Russland) - ca. } 1.200\end{array}$ \\
\hline $\begin{array}{l}\text { Juni } \\
2004\end{array}$ & $\begin{array}{l}\text { Pawlogradwu- } \\
\text { hillja }(92.1 \%)\end{array}$ & $\begin{array}{l}\text { Kohleberg- } \\
\text { bau }\end{array}$ & $\begin{array}{l}\text { Investitionszusage von } \\
0,1 \text { Mio. US\$ sowie } \\
\text { weitere Auflagen }\end{array}$ & $\begin{array}{l}\text { Awdejewskij KChZ [SCM] - } 263 \\
\text { Ispat Ugol }\end{array}$ \\
\hline $\begin{array}{l}\text { Juli } \\
2004\end{array}$ & $\begin{array}{l}\text { Eisenerzwerk } \\
\text { Ingulezkyj } \\
(37,57 \%)\end{array}$ & $\begin{array}{l}\text { Metallin- } \\
\text { dustrie }\end{array}$ & & Smart-Group (Russland) - 31 \\
\hline $\begin{array}{l}\text { Nov. } \\
2004\end{array}$ & $\begin{array}{l}\text { Krasnodonwu- } \\
\text { hillja }(60 \%)\end{array}$ & $\begin{array}{l}\text { Kohle- } \\
\text { bergbau }\end{array}$ & $\begin{array}{l}\text { Investitionszusage über } \\
188 \text { Mio. US\$ und } \\
\text { weitere Auflagen }\end{array}$ & Awdejewskij KChZ [SCM] - 263 \\
\hline
\end{tabular}


Tabelle 2: Die großen ukrainischen Privatisierungsauktionen 2000 - 2005 (Fortsetzung)

\begin{tabular}{|c|c|c|c|c|}
\hline Datum & $\begin{array}{l}\text { Verkaufter } \\
\text { Aktienanteil }\end{array}$ & Branche & Bedingungen & $\begin{array}{l}\text { Bieter - Gebot [in Mio. US\$] } \\
\text { [der erfolgreiche Bieter ist fett hervorgehoben] }\end{array}$ \\
\hline $\begin{array}{l}\text { Juli } \\
2004\end{array}$ & $\begin{array}{l}\text { Eisenerzwerk } \\
\text { Zentralnyj } \\
(50 \%)\end{array}$ & $\begin{array}{l}\text { Metall- } \\
\text { industrie }\end{array}$ & & $S C M-20$ \\
\hline $\begin{array}{l}\text { Juli } \\
2004\end{array}$ & $\begin{array}{l}\text { Eisenerzwerk } \\
\text { Piwnitschnyj } \\
(50 \%)\end{array}$ & $\begin{array}{l}\text { Metall- } \\
\text { industrie }\end{array}$ & & Artanik $[\mathrm{SCM}]-39$ \\
\hline $\begin{array}{l}\text { Mai } \\
2005\end{array}$ & $\begin{array}{l}\text { Krasnodonwu- } \\
\text { hillja }(40 \%)\end{array}$ & $\begin{array}{l}\text { Kohle- } \\
\text { bergbau }\end{array}$ & & Avdeevskij KKhZ [SCM] - 98 \\
\hline $\begin{array}{l}\text { Okt. } \\
2005\end{array}$ & $\begin{array}{l}\text { Kryworishstal } \\
(93,02 \%)\end{array}$ & $\begin{array}{l}\text { Metall- } \\
\text { industrie }\end{array}$ & Strategischer Investor & $\begin{array}{l}\text { Mittal Steel Germany (Deutschland/Indien) }-\mathbf{4} 790 \\
\text { Industrial Group [Arcelor + ISD] } \\
\text { Smart-Group (Russland) }\end{array}$ \\
\hline
\end{tabular}

Anmerkung: Alle Auktionen mit einem Wert von über 10 Mio. US\$. Die erfassten Auktionen hatten einen Anteil von über $80 \%$ an den staatlichen Privatisierungseinnahmen im Zeitraum von 2000 bis 2005.

Quellen: Ukrainischer Fonds für Staatsvermögen (uww.spfugov.ua), Institute for Economic Research and Policy Consulting

(www.ier.kiev.ua), NewsBase-Archive (www.newsbase.com), BBC World Monitoring.

\section{Dokumentation}

\section{Schleuderpreise?}

Um abschätzen zu können, wieweit der Verkaufspreis manipuliert wurde, ist es erforderlich, den tatsächlichen Wert des privatisierten Unternehmens zu bestimmen. In der Aktienanalyse ist hierbei das Kurs-Gewinn-Verhältnis (KGV) ein zentraler Indikator. Es wird berechnet als Quotient aus Aktienkurs und anteiligem Unternehmensgewinn. Das KGV gibt damit für eine Aktie an, mit dem Wievielfachen des jährlichen Unternehmensgewinns sie bewertet wird. Derselbe Indikator kann auch für die bei Privatisierungsauktionen verkauften Unternehmensanteile gebildet werden. Das Preis-Gewinn-Verhältnis, das im Folgenden der Einfachheit halber auch KGV genannt werden soll, gibt dann an, das Wievielfache des jährlichen Unternehmensgewinns (Nettogewinn nach Steuern) vom Käufer bezahlt werden musste. Ein KGV von 10 bedeutet so, dass der Käufer anteilig 10 Jahresgewinne bezahlen muss, sich seine Investition also - unter der Annahme gleich bleibender Gewinne - nach 10 Jahren amortisiert hat.

Um den Verkaufspreis für die privatisierten Unternehmen einzuschätzen, werden deshalb auf der Grundlage des KGV fünf Kategorien gebildet: sehr günstig, günstig, normal, ungünstig und negativ. Sehr günstig beschreibt dabei ein KGV von 1 bis 3, d.h. dass der Investor spätestens nach drei Jahren den Kaufpreis aus seinem Anteil am Gewinn zurückerhalten hat. Landes- und branchenunabhängig kann ein entsprechender Verkaufspreis als extrem vorteilhaft für den Käufer betrachtet werden. Hier liegt ein Manipulationsverdacht nahe. Ungünstig beschreibt ein KGV, das über 40 liegt. Auch hier kann die Bewertung landes- und branchenunabhängig erfolgen. Negativ ist ein KGV, wenn das entsprechende Unternehmen Verluste ausweist. In beiden Fällen gibt das KGV keine Anzeichen für einen zu niedrigen Preis. Zum Vergleich: Das durchschnittliche KGV der börsennotierten Unternehmen in Osteuropa (EMEastern Europe) lag 2004 bei 8, das KGV der größten börsennotierten europäischen Unternehmen (Euro Stoxx 50) lag 2004 bei 14 .

Das KGV ist jedoch kein eindeutiger Indikator für den Unternehmenswert. Erstens sind die Gewinne teilweise erheblichen Schwankungen unterworfen. Die mit der Privatisierung verbundenen Änderungen in der Unternehmensleitung etwa können auch zu einem schlechten Jahr mit entsprechendem Gewinnrückgang führen. Um diesen Effekt abzuschwächen, wurde hier für den Gewinn ein Durchschnittswert aus zwei Jahren gebildet. Zweitens ist der Gewinn aber kein eindeutiger Indikator für den Unternehmenswert. Zum einen besitzen auch mit Verlust arbeitende Unternehmen aufgrund ihres Vermögens einen Wert. Zum anderen hängt der Wert eines Unternehmens nicht nur von den gegenwärtigen Gewinnen ab, sondern sehr stark auch von der Erwartung bezüglich zukünftiger Gewinne. Der Unternehmenswert erhält damit eine subjektive Komponente. 


\begin{tabular}{|c|c|c|c|c|c|c|c|c|c|c|c|c|c|c|c|c|c|c|}
\hline 0 & 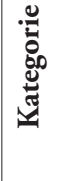 & 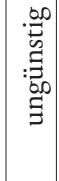 & 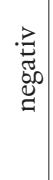 & 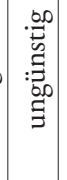 & 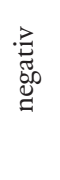 & 胥 & 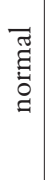 & 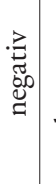 & 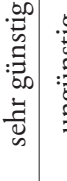 & 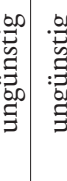 & 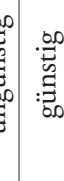 & 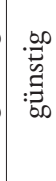 & 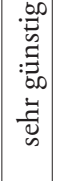 & 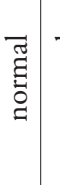 & 胥 & 胥 & & \\
\hline & 壳 & $\hat{n}$ & $\stackrel{Y}{i}$ & $\underset{\widetilde{\sim}}{3}$ & $\underset{\underset{I}{\longrightarrow}}{\stackrel{\rightleftarrows}{ }}$ & $\stackrel{0}{\longrightarrow}$ & $\stackrel{m}{=}$ & $\begin{array}{l}0 \\
1\end{array}$ & $N=$ & $\vec{F} \quad \nabla$ & $\Rightarrow n$ & 6 & $m$ & \pm & 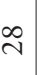 & के & & -7 \\
\hline
\end{tabular}

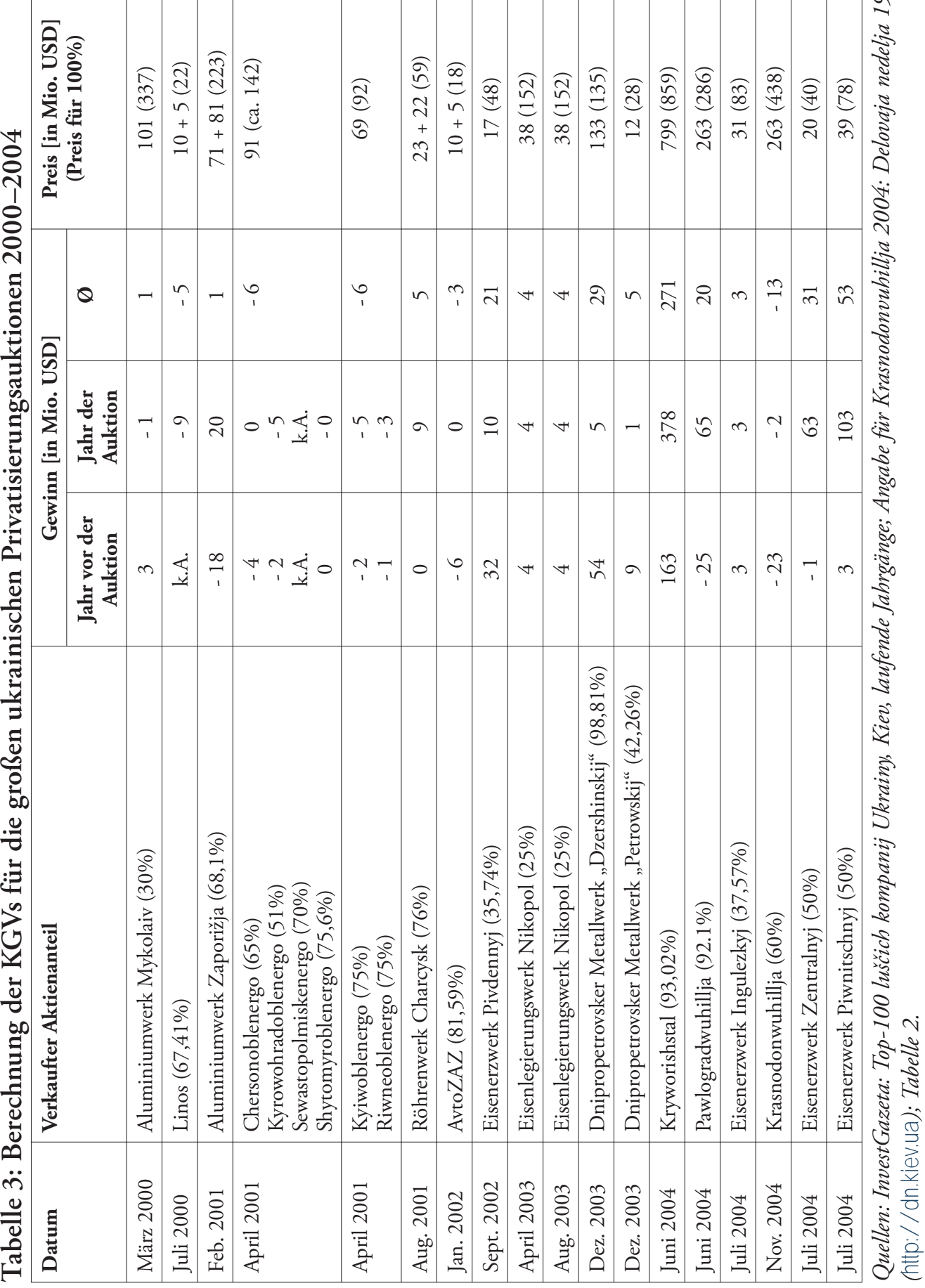




\section{Portraits}

\section{Die an Privatisierungsauktionen beteiligten Holdings}

\section{Industrial Union of Donbas (Industrialnij Sojuz Donbassa - ISD)}

Die Industrial Union of Donbas (Industrialnij Sojuz Donbassa - ISD) wurde 1995 von regionalen Eliten in Donezk gegründet. In den folgenden Jahren gelang es ihr die Kontrolle über die Erdgasversorgung der Region Donezk zu übernehmen. Seit 1998 benutzte ISD die resultierenden Finanzströme, um sich in der Metallindustrie zu engagieren. Sie erwarb Stahlproduzenten in der gesamten Ostukraine und begann auch sich in der Eisenerz- und Kohleproduktion zu engagieren. Im Rahmen einer umfassenden Investitionsstrategie übernahm ISD die Kontrolle über die gesamte Produktionskette der Stahlindustrie und stieg auch in den Maschinenbau ein. ISD erwarb zusätzlich Beteiligungen in der Lebensmittelindustrie, dem Transportwesen und der Gastronomie.

ISD wird nach außen durch ihren Leiter Sergei Taruta präsentiert. Die tatsächliche Eigentümerstruktur der Holding ist aber unklar. Als offizielle Eigentümer fungieren fünf staatliche und private Strukturen, darunter z.B. die Akademie der Wissenschaften der Ukraine und die Donezker Industrie- und Handelskammer.

\section{Interpipe}

Der Aufstieg der Holding Interpipe begann 1997. Sie erwarb wie die meisten Holdings Unternehmensbeteiligungen in den unterschiedlichsten Branchen. Der Schwerpunkt des Engagements lag aber eindeutig in der Metallindustrie und insbesondere in der Röhrenproduktion. Im Medienbereich wurden Beteiligungen an drei nationalen Fernsehsendern sowie einer Tageszeitung erworben. Die Holding mit Sitz in Dnipropetrowsk wird von Viktor Pintschuk kontrolliert. Pintschuk, der Schwiegersohn des ehemaligen Präsidenten Leonid Kutschma, war von 2000 bis 2004 auch offizieller Berater des Präsidenten. Er hat enge Verbindungen zu Sergei Tihipko, der von 1997 bis 2001 in der Regierung für Wirtschaftsfragen zuständig war und anschließend bis 2004 die Leitung der Zentralbank übernahm.

\section{Privatbank}

Die Privatbank wurde 1992 von Sergei Tihipko in Dnipropetrowsk gegründet. Durch Finanzspekulationen und mit Unterstützung der Zentralbank konnte sie eine der erfolgreichsten Banken in der Ukraine werden. Mit dem Wechsel von Tihipko in die Politik und seiner Allianz mit Interpipe wurde die Privatbank von Igor Kolomojskij übernommen. Im Zuge der Privatisierungen wuchs die Holding der Bank zu einer der größten der Ukraine. Sie konzentrierte ihre Beteiligungen auf die Metallindustrie und die Erdölindustrie, erwarb aber auch Firmen in etlichen anderen Branchen wie der chemischen Industrie, der Lebensmittelindustrie und dem Baubereich.

\section{System Capital Managemen (SCM)}

System Capital Managemen (SCM) fasst seit 2000 die Unternehmensbeteiligungen von Rinat Achmetow zusammen. Achmetow übernahm 1995 die Handelsfirmen des Donezker Unternehmers Ahat Bragin, nachdem dieser einem Attentat zum Opfer gefallen war. In den folgenden Jahren gelang Achmetow die Integration in die neue Elite der Region Donezk. In enger Kooperation mit der Industrial Union of Donbas erwarb er bedeutende Firmen der Metallindustrie. Zusätzlich engagierte er sich in der Lebensmittelindustrie, der Gastronomie und dem Finanzsektor. Ihm gehört auch der Fußballverein Schachter Donezk. Bei den Parlamentswahlen im März 2006 erhielt Achmetow über die Liste der Partei der Regionen einen Abgeordnetensitz. 


\section{Chronik}

\section{Vom 9. bis 22. Mai 2006}

\begin{tabular}{|c|c|}
\hline 9.5 .2006 & $\begin{array}{l}\text { Bei den Feierlichkeiten zum Jahrestag des Sieges über das faschistische Deutschland ruft Präsident } \\
\text { Viktor Juschtschenko erneut zur Versöhnung zwischen den Veteranen der Roten Armee und den na- } \\
\text { tionalistisch orientierten Veteranen der UPA auf. Er schlägt vor, die Anerkennung der UPA-Kämpfer } \\
\text { als Kriegsveteranen in die laufenden Koalitionsverhandlungen aufzunehmen. Der Vorsitzende der an } \\
\text { den laufenden Koalitionsverhandlungen beteiligten Sozialisten, Alexander Moros, lehnt die Themati- } \\
\text { sierung dieser Frage ab. }\end{array}$ \\
\hline 10.5 & $\begin{array}{l}\text { Präsident Viktor Juschtschenko unterschreibt einen Erlass, der die Wehrdienstzeit verkürzt. Die Regel- } \\
\text { dienstzeit bei den Bodentruppen wird von } 18 \text { auf } 12 \text { Monate verkürzt. Die Dienstzeit für Universitäts- } \\
\text { absolventen wird von } 12 \text { auf } 9 \text { Monate gesenkt. }\end{array}$ \\
\hline 11.5 .2006 & $\begin{array}{l}\text { Die Regierung entlässt den Leiter der staatlichen Energieholding Naftohaz Ukrainy, Alexander Iwt- } \\
\text { schenko, da dieser als Abgeordneter ins Parlament wechselt. }\end{array}$ \\
\hline 12.5 .2006 & $\begin{array}{l}\text { In einem Interview mit der polnischen Gazeta Wyborcza spricht sich Präsident Viktor Juschtschenko } \\
\text { dezidiert gegen eine Regierungskoalition mit der Partei der Regionen von Viktor Janukowitsch aus. Er } \\
\text { schließt nicht aus, dass Julia Timoschenko erneut Ministerpräsidentin wird. }\end{array}$ \\
\hline 13.5 .2006 & $\begin{array}{l}\text { Bei einem Besuch im polnischen Dorf Pawlokoma rufen der polnische Präsident Lech Kaczynski und } \\
\text { der ukrainische Präsident Viktor Juschtschenko zur Versöhnung über die gemeinsame Geschichte auf. } \\
\text { Der Besuch erfolgte aus Anlass der Enthüllung eines Denkmals für ukrainische Dorfbewohner, die } \\
1945 \text { von polnischen Militärs ermordet worden waren. }\end{array}$ \\
\hline 16.5 . & $\begin{array}{l}\text { Die an den Koalitionsverhandlungen zur Regierungsbildung beteiligten Parteien haben nach einem } \\
\text { Bericht von Radio Free Europe / Radio Liberty eine ganze Reihe unterschiedlicher Entwürfe für Ko- } \\
\text { alitionsverhandlungen erstellt. Roman Zwaritsch, einer der Verhandlungsführer von Unsere Ukraine, } \\
\text { erklärt, seine Partei würde die Verhandlungen aussetzen, da die Posten des Ministerpräsidenten und des } \\
\text { Parlamentsvorsitzenden an die beiden anderen Verhandlungspartner gehen sollen. }\end{array}$ \\
\hline 16.5 .2006 & $\begin{array}{l}\text { Im seit } 1997 \text { andauernden Konflikt um die Meeresgrenze mit Rumänien reicht die Ukraine ein Me- } \\
\text { morandum beim Internationalen Gerichtshof in Den Haag ein. Die Ukraine betrachtet die Schlangen- } \\
\text { inseln als ihr Territorium, das einen Anspruch auf das umgebende Seeterritorium im Schwarzen Meer } \\
\text { begründet. Rumänien hält die Inseln nur für eine Felsgruppe und beansprucht das Gebiet als eigenes } \\
\text { Küstengewässer. }\end{array}$ \\
\hline $\begin{array}{l}22 .- \\
23.5 .2006\end{array}$ & $\begin{array}{l}\text { Die Präsidenten der GUAM-Staaten (Georgien, Ukraine, Aserbaidschan, Moldova) treffen sich in } \\
\text { Kiew. Zentrale Themen der Gespräche sind die Diversifizierung von Energieimporten und der Um- } \\
\text { gang mit separatistischen Enklaven. }\end{array}$ \\
\hline
\end{tabular}

Die Ukraine-Analysen werden mit Unterstützung durch die Otto-Wolff-Stiftung gemeinsam von der Forschungsstelle Osteuropa an der Universität Bremen und der Deutschen Gesellschaft für Osteuropakunde herausgegeben.

Die Meinungen, die in den Ukraine-Analysen geäußert werden, geben ausschließlich die Auffassung der Autoren wieder. Abdruck und sonstige publizistische Nutzung sind nach Rücksprache mit der Redaktion gestattet. Redaktion und technische Gestaltung: Matthias Neumann, Heiko Pleines Ukraine-Analysen-Layout: Cengiz Kibaroglu, Matthias Neumann ISSN 1862-555X (c) 2006 by Forschungsstelle Osteuropa, Bremen

Forschungsstelle Osteuropa • Publikationsreferat • Klagenfurter Str. 3 - 28359 Bremen • Telefon: +49 421-218-7891 • Telefax: +49 421-218-3269 e-mail: publikationsreferat@osteuropa.uni-bremen.de • Internet-Adresse: www.ukraine-analysen.de 